\title{
COLD PLASMA CHEMISTRY AND DIAGNOSTICS
}

\author{
Isabel Tanarro,* Víctor J. Herrero, E. Carrasco, M. Jiménez-Redondo \\ Inst. Estructura de la Materia, CSIC \\ Serrano 123, 28006 Madrid, Spain \\ itanarro@iem.cfmac.csic.es
}

\begin{abstract}
$\underline{\text { Abstract }}$
This paper gives an overview of the main chemical processes which take place in cold plasmas generated in glow discharges, both in the gas phase and at the surfaces in contact with the plasma. Illustrative examples are provided where the relevance of different processes is evinced. Most common plasma characterization techniques and their recent improvements are also presented briefly.
\end{abstract}

\section{$\underline{\text { Keywords }}$}

Cold plasma, glow discharge, plasma chemistry, plasma kinetics, plasma diagnostics, plasmasurface interaction.

*Corresponding author 


\section{Introduction}

Cold plasmas, produced mainly in electric glow discharges, are of great interest in a large number of scientific and technological fields [1-3]. In cold plasmas, ionization and dissociation of molecular precursors, initiated by collisions between free electrons and gas phase species, are followed by a great variety of processes that lead to special chemical conditions, very suitable for thin film growth, surface conditioning, biomaterials disinfection, energy saving lamps, laser sources, etc [4]. Glow discharges can also simulate the border conditions of plasmas generated in fusion reactors [5]. In addition, they are very useful to produce radicals, ions and excited states, unstable under ordinary conditions, whose study can contribute to the understanding of the generation of similar compounds in the interstellar space and in the ionospheres of some planets [6].

The complex kinetics of cold molecular plasmas is difficult to disentangle, since they host a great deal of different species, with characteristic concentrations and lifetime scales spanning many orders of magnitude. Understanding the very diverse processes involved in these plasmas, both in homogeneous and heterogeneous reactions, needs basic reference data, including cross sections, rate coefficients, sticking probabilities and so on, often unavailable. Therefore, a great effort and a variety of experimental and modelling techniques are currently devoted to the characterization of these systems.

Experimental diagnosis of glow discharges relies mainly on the use of mass spectrometry, spectroscopic techniques and electric probes [4,7]. The development of particular methods for the sensitive detection of transient species, which play a key role in the plasma chemistry, is an active research field. Comparison of experimental results with theoretical models allows a better understanding of these systems and, as a result, an improvement in their applications.

In this work, the main kinetic processes taking place in cold plasmas generated in glow discharges and the methods most frequently used for plasma diagnostic will be 
discussed. Initially, we will see the processes that lead to plasma generation and the mechanisms that follow plasma ignition, largely responsible for its high chemical reactivity, even at room temperatures. Then, a short description of the experimental techniques presently available to study the different species involved is included. Finally, some cases, based mostly on our own work, will exemplify the relevance of different key processes.

\section{Cold Plasma Chemistry}

\section{$\underline{\text { 2.1. Plasma generation in glow discharges }}$}

Cold plasmas are plasmas with a low ionization degree, far from thermal equilibrium, where the electron temperatures $\left(T_{e}\right)$ are much higher than the gas temperatures $\left(T_{\text {gas }}\right)$. They show high stability and occur most commonly at low pressures.

Glow discharges are produced either by direct current, radiofrequency or microwave sources, where free electrons are accelerated and gain kinetic energy efficiently from the electromagnetic field. This energy is exchanged easily through elastic collisions with other electrons, giving rise typically to Maxwellian-like electron energy distributions (EED). However, the proportion of energy transferred from one electron to a much heavier ion or neutral in each elastic collision is very low, smaller than four times the mass ratio between the electron and the other body. The average kinetic energy transfer increases with the number of collisions, i.e. with growing gas pressure.

Within the high energy tail of the EED, those electrons with higher energies than the ionization threshold of the precursors can ionize them, liberating new free charges that compensate wall losses and neutralizations in the gas phase. These additional charges are essential to maintain the plasma stability. Glow discharges are stable over relatively broad pressure ranges, although the precise limits depend on the characteristic dimensions of the discharge. At the lowest pressures, electrons might gain much energy between collisions and enhance their ionization capability, but their collisions with neutrals are too scarce to generate 
sufficient new free charges, and the plasma extinguishes. At the highest pressure limits, the average gain of electron energy between collisions is not enough to compensate previous energy losses and to reach the ionization threshold. Then, the plasma becomes unstable and inhomogeneous, with tendency to arching.

\subsection{Main gas phase reactions}

Besides ionization, primary processes by electron impact on atoms and molecules are molecular dissociation and excitation to upper energy levels. Ionizations and dissociations require threshold energies of $\sim 5-20 \mathrm{eV}$. Above these thresholds, the cross sections increase with electron energy up to $\sim 50-100 \mathrm{eV}$, decreasing smoothly afterwards. The electron velocity, times the cross section, integrated along the whole velocity range, gives the rate coefficient, $k$, for each reaction. Figure 1 displays the $k$ dependences on $T_{e}\left(k=a \times k_{B} T_{e}^{b} \times e^{-}\right.$ $c / k_{B} T_{e}$ ), for some rate coefficients involved in $\mathrm{H}_{2}$ plasmas [8], assuming Maxwellian EED. For $k_{B} T_{e} \sim 2-8 \mathrm{eV}$, typical of glow discharges, $k \sim 10^{-10}-10^{-9} \mathrm{~cm}^{3} \mathrm{~s}^{-1}$ are commonly found. The electron densities, $N_{e}$, span usually a range from $10^{9}$ to $10^{11} \mathrm{~cm}^{-3}$; this means that, without any recovery process, precursors would be totally ionized or dissociated within characteristic times $\left(\tau=k \times N_{e}\right)$ of tenths of seconds.

Excitations require discrete energy values of some $\mathrm{eV}$ to populate electronic upper levels, and tenths or hundredths of $\mathrm{eV}$ for vibrational or rotational excitations, respectively. Spontaneous emission from excited levels provides one of the most characteristic properties of plasmas: light radiation, and allows the identification of the species through their spectral analysis.

Selective losses of electron energies by ionization, dissociation and excitation can change the EED and move it away from a Maxwellian distribution. 


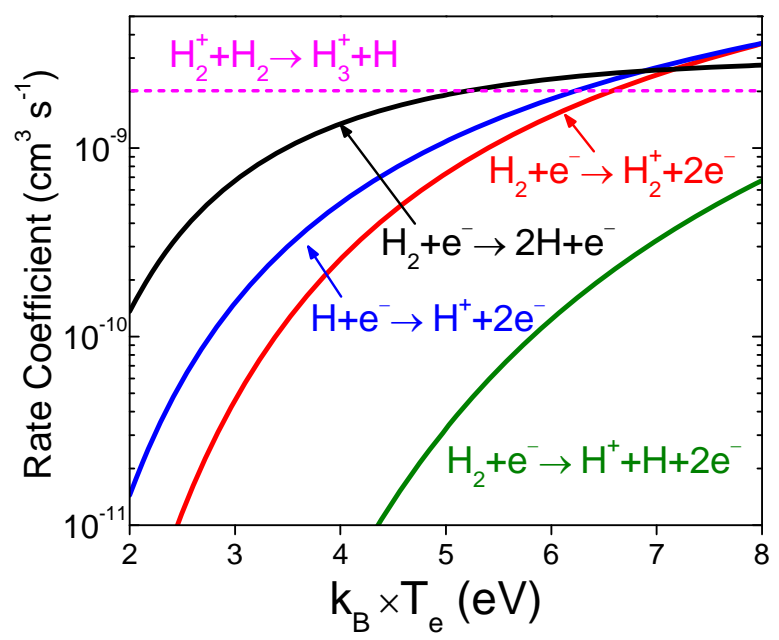

Figure 1. Rate coefficients for relevant homogeneous reactions in $\mathrm{H}_{2}$ plasmas, taken from the expressions given in [8].

Ions, atoms and radicals in ground or excited states liberated by the former processes are extremely reactive species, able to undergo secondary reactions at room temperature, which efficiently transform the plasma precursors into other products [9], or interact with the surrounding walls, changing the surface conditions. Their lifetimes in the plasma are very short, roughly, $\sim 10^{-3}-10^{-2} \mathrm{~s}$ for atoms and radicals and $\sim 10^{-7}-10^{-6} \mathrm{~s}$ for ions [10], since they recombine or neutralize very quickly; and their steady state concentrations are several orders of magnitude lower than those of stable species (Figure 2). One exception to this rule is that the concentration of radicals and atoms can be unusually high in discharge chambers made from materials with a low coefficient for heterogeneous surface recombination [11-13]. The lifetimes of excited states extend $\sim 10^{-3}-10^{-9} \mathrm{~s}$, depending on their transition probabilities and quenching cross sections. Long lived excited states display higher populations than those which decay faster, leading to partial temperatures of some thousands of Kelvin (assuming Boltzmann distributions). Ions at the plasma sheath are accelerated in the electric field between the glow and the reactor surfaces, gaining energies up to tens of $\mathrm{eV}$ in $\mathrm{RF}$ discharges with dominant inductive coupling (high power, $\mathrm{H}$ mode) or MW discharges, and hundreds of 
$\mathrm{eV}$ in RF discharges with dominant capacitive coupling (low power, E mode) or, as well, when reaching a grounded cathode in DC reactors. These energies enhance markedly the effectiveness of ion collisions on the surfaces.

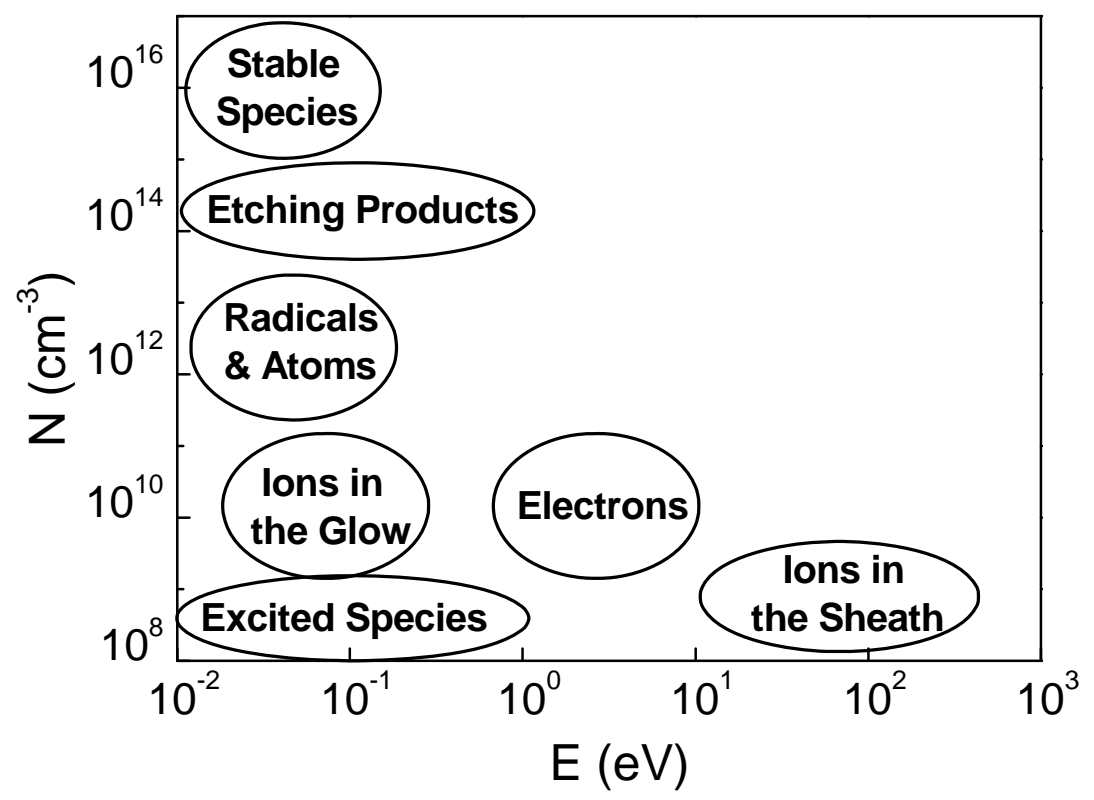

Figure 2. Typical concentrations and energies of different species generated in glow discharges.

In gas phase, bimolecular reactions with no energy barrier between transients and stable molecules predominate largely over other reactions. Their cross sections decrease typically with collision energy as $\mathrm{E}^{-1 / 2}$ (Langevin behaviour), but the relative velocity between the colliding particles is proportional to $\mathrm{E}^{1 / 2}$. Then, the resulting rate coefficients are independent on $T_{\text {gas }}$, often with values $k>10^{-10} \mathrm{~cm}^{3} \mathrm{~s}^{-1}$ for reactions involving radicals, and $k>10^{-9} \mathrm{~cm}^{3} \mathrm{~s}^{-1}$ for ions. For example, $k=2 \times 10^{-9} \mathrm{~cm}^{3} \mathrm{~s}^{-1}$ for the process $\mathrm{H}_{2}{ }^{+}+\mathrm{H}_{2} \rightarrow \mathrm{H}_{3}{ }^{+}+\mathrm{H}$ (Figure 1). Electron impact dissociative neutralizations like $\mathrm{H}_{3}{ }^{+}+\mathrm{e}^{-} \rightarrow 3 \mathrm{H}\left(\right.$ or $\mathrm{H}_{2}+\mathrm{H}$ ) have even larger coefficients $\left(k \sim 10^{-7}-10^{-8} \mathrm{~cm}^{3} \mathrm{~s}^{-1}\right)$, and can be very important in space chemistry; nevertheless, they are irrelevant in glow discharges as compared with wall neutralizations. 
In bimolecular reactions with an energy barrier, the rate coefficients decrease exponentially with $T_{\text {gas }}$ (Arrhenius' like dependence) and display small values $\left(k<10^{-12} \mathrm{~cm}^{3} \mathrm{~s}^{-}\right.$ ${ }^{1}$ ), even at some thousands of Kelvin. So, although usually important in chemical reactors at high temperatures, they can be disregarded in cold plasma kinetics. Exothermic association reactions like $\mathrm{H}+\mathrm{H} \rightarrow \mathrm{H}_{2}$ are not possible without a third body, which takes away the excess energy of molecular formation. However, gas phase reactions among three bodies exhibit extremely low probabilities at the low pressures characteristic of glow discharges.

\subsection{Surface reactions}

Glow discharges interact with the surrounding surfaces, primarily through charge neutralization and secondary electron emission, both essential to maintain the ionization degree of the plasma, especially in DC discharges; and also through heterogeneous recombination, which returns stable species to the discharge, often different from the precursors. For example, air discharges can produce nitrogen oxides by $\mathrm{N}$ and $\mathrm{O}$ wall recombination, depending on the surface material [14-17]. The interacting surfaces may be those of the discharge reactor, samples introduced for processing, or even correspond to small particles in suspension, generated in the plasma. Heterogeneous recombination of radicals or atoms can take place through different intermediate processes, like physisorption, chemisorption, thermal desorption, surface diffusion, and both, Eley-Rideal and LangmuirHinshelwood mechanisms [18].

In addition, some material proceeding from the discharge can be deposited on the walls, and, inversely, the surfaces can be eroded, releasing diverse components to the plasma. In general, these phenomena can change completely the properties of the exposed surfaces, leading to many applications, especially relevant when the materials to be processed do not tolerate the high temperatures needed in most chemical processes. Particular attention has 
been devoted to the plasma enhanced chemical vapour deposition (PECVD) of amorphous hydrogenated silicon (a-Si:H) [19,20], carbon (a-C:H) [21-23], and nitride films [24-26].

Although radicals are usually the dominant reactive species in the weakly ionized plasmas of glow discharges, ions can also influence the surface processes in several important ways, specially at the lowest pressures and highest powers. Ions contribute to film growth either by direct implantation or by creating reactive sites on the surfaces; and they also cause erosion by physical or chemical sputtering. The most abundant transient species in the gas phase do not necessarily play a key role in these processes. For instance, in PECVD, actual growth precursors have typically high sticking coefficients and very low concentrations. Thus, the search for these precursors requires the use of very sensitive detection techniques.

\section{Diagnostic methods}

The main challenge in plasma diagnostics is to detect, identify and quantify the key species in plasma processing, obtaining data such as their energy contents, concentrations, rate coefficients of the reactions involved, lifetimes, geometrical distributions, and so on. A whole diagnostic allows an understanding of the main processes responsible for the plasma behaviour, frequently with assistance of kinetic models [27]. The number of plasma species can be very large, their concentrations spanning several orders of magnitude, as well as their energies (figure 2). Moreover, unstable species disappear quickly, once the discharge is turned off, and must be studied "in situ”.

The methods most commonly employed rely on mass spectrometry, spectroscopy in different electromagnetic regions and electrical probes $[1-3,7,28]$. Some techniques are suitable to study many different species, but lack the sensitivity to detect the minor ones; whereas more specific and powerful procedures are restricted to particular species. Therefore, several complementary techniques should be used for a quite complete plasma diagnostics. On the other hand, experimental plasma reactors are commonly designed to facilitate their 
characterization with the most appropriate set-ups, however, in industrial reactors, the access to the plasma is often very restricted.

Being intrinsically light-emitting sources, plasmas can be characterized first of all by emission spectroscopy [29,30]. This non intrusive technique has been used traditionally to identify excited species with high selectivity and sensitivity, and to determine their temperatures; although absolute concentrations, relying on complex data such as excitation cross-sections and transition probabilities, are quite difficult to estimate. Actinometry allows the determination of relative concentrations of diverse species, as compared with a reference (most commonly, a noble gas) introduced at very low concentration, by comparing the intensities of specific lines which have similar excitation cross section dependences with electron energy [31,32]. Dispersive instruments are employed for visible and ultraviolet (UV) emissions, and Fourier transform spectrometers are more advantageous for the infrared (IR) region, where the background radiation is much larger than that in the visible and UV ranges, and the detectors less sensitive [30,33]. Light emitting sources like Globars and blackbodies in the IR and visible, or cyclotrons and deuterium lamps in the UV, allow the use of the former instruments for broadband absorption spectroscopy. Absolute concentrations of the species can be quite directly inferred from absorbance measurements, as well as gas temperatures. Tuneable high resolution laser sources available at present, combined with optical multi-pass configurations, are very advantageous for absorption studies of species at very low densities, or when the transitions of different species are very close to each other [33-35]. High power pulsed lasers have allowed the development of very sensitive and selective techniques, such as laser induced fluorescence and multi-photon ionization [36].

Mass spectrometry, most often with quadrupole mass spectrometers based on ionization by electron impact and detection of ions according to their mass/charge ratio, is a versatile and well established technique to detect major neutrals [7,37]. These species are extracted without plasma perturbation through a small diaphragm or a fine valve towards the 
spectrometer, located in a differentially pumped vacuum chamber. However, dissociative ionization of molecules gives rise to characteristic fragmentation patterns that may cause overlapping of the mass peaks of different parent molecules and make the identification of species difficult $[23,38]$, in spite of the numerical methods presently available to extract this information [39] or the use of precursors with different isotopic composition. Distinguishing among stable species by their condensation temperatures in programmed desorption-mass spectrometry [40] has improved recently the technique in particular cases.

Detection of radicals is specially hampered by the overlapping of mass peaks proceeding from the much larger signals of stable species. Ion attachment mass spectrometry with light ions, like $\mathrm{Li}^{+}$[41], and the use of electron energies below the dissociative ionization threshold are useful alternatives to prevent fragmentations. They have presently made the detection of radicals at very low concentrations possible, with direct sampling $[42,43]$, or even employing molecular beams [44,45]. Plasma ions can be extracted directly from the discharge and focused on the mass filter of the spectrometer, without further electron-impact ionization, by using electrostatic lenses available in current plasma monitoring mass spectrometers. These spectrometers also have energy analyzers that lately have allowed the identification of the ions by their energy distributions [46,47].

Electron temperature, charge density and plasma potential are traditionally measured with Langmuir probes inserted into the plasma [2,7,48,49]. Fiber-optic catalytic probes of various materials have been recently developed to measure dissociation degrees of pure gases such as $\mathrm{H}_{2}, \mathrm{O}_{2}$ or $\mathrm{N}_{2}$ in plasma afterglows [50,51]. New calorimetric probes allow estimations of energy fluxes on substrates [52]. In all these cases, special care should be taken to minimize perturbations of the plasma [53-55]. Electron temperatures can be deduced alternatively from optical emission measurements assisted by collisional radiative models [56,57]. The emission can proceed directly from the glow or, sometimes, from the excitation of molecular beams introduced as test probes [58-60]. 
Modulation procedures allow extending several of the former techniques to time resolved and phase sensitive plasma studies [33,35,44,45], increasing by orders of magnitude the sensitivity for the detection of minor species, and adding valuable information on reaction coefficients and sticking coefficients.

Dust particles formed in plasmas are receiving increasing attention. Their density and size growth can be monitored by light scattering [61]. Surface analysis requires additional techniques, out of the scope of this work.

\section{Examples of cold plasma chemistry}

$\mathrm{H}_{2}$ glow discharges are the simplest example of a molecular plasma. Electron impact ionization and dissociation of $\mathrm{H}_{2}$ lead to the primary ions $\mathrm{H}_{2}{ }^{+}$and $\mathrm{H}^{+}$, and $\mathrm{H}$ atoms. $\mathrm{H}_{3}{ }^{+}$is then produced through the reaction $\mathrm{H}_{2}{ }^{+}+\mathrm{H}_{2} \rightarrow \mathrm{H}_{3}{ }^{+}+\mathrm{H}$ and, as pressure grows, becomes even the major ion [8,10]. Fig.3-a shows the different relative ion concentrations in a $\mathrm{H}_{2}$ discharge at 0.8 and $2 \mathrm{~Pa}$. With growing pressure, $T_{e}$ decreases, and the rate coefficient for $\mathrm{H}_{2}$ ionization becomes smaller than that for $\mathrm{H}_{3}{ }^{+}$formation (Fig.1), leading to a very efficient transformation of $\mathrm{H}_{2}{ }^{+}$in $\mathrm{H}_{3}{ }^{+} . \mathrm{H}_{3}{ }^{+}$is a key species for the generation of more complex molecules in the universe, like water [6], and since the 1980's, it has been increasingly detected in interstellar clouds [62], in spite of the low temperatures $\left(\mathrm{T}_{\text {gas }} \sim 10-20 \mathrm{~K}\right)$ in these regions. In general, hydrogen containing plasmas like $\mathrm{N}_{2}+\mathrm{H}_{2}, \mathrm{Ar}+\mathrm{H}_{2}, \mathrm{CH}_{4}+\mathrm{H}_{2}$, etc., exhibit also remarkable concentrations of protonated ions $\left(\mathrm{N}_{2} \mathrm{H}^{+}, \mathrm{ArH}^{+}, \mathrm{CH}_{5}{ }^{+} \ldots\right)$, that can often exceed those of their non-protonated counterparts [38,63].

Heterogeneous processes in $\mathrm{H}_{2}$ discharges lead to the neutralization of ions, and to $\mathrm{H}$ adsorption, which then recombines with impinging $\mathrm{H}$ atoms and returns $\mathrm{H}_{2}$ to the plasma. Glow discharges of $\mathrm{H}_{2}+\mathrm{D}_{2}$ mixtures evince better the recombination of hydrogen in the walls, through the efficient formation of HD, distinguishable from the precursors. 
Surface reactions are also responsible for the formation of nitrogen oxides in $\mathrm{N}_{2}+\mathrm{O}_{2}$ discharges [14,15,17], and ammonia in $\mathrm{N}_{2}+\mathrm{H}_{2}$ discharges [64]; with efficiencies that depend on the reactor materials and surface conditions, but show higher production rates in stainless steel reactors than in Pyrex or quartz. Fig. 3-b displays the signal obtained in a $0.4 \mathrm{~Pa}$ air discharge, yielding a NO concentration even higher than that of $\mathrm{O}_{2}$ [15].

The chemical erosion of carbon films by $\mathrm{He}+\mathrm{O}_{2}$ plasmas is deduced in Fig.3.c from the $\mathrm{O}_{2}$ depletion and the $\mathrm{CO}, \mathrm{CO}_{2}$ and $\mathrm{H}_{2}$ generation in $\mathrm{He}+20 \% \mathrm{O}_{2}, 2 \mathrm{~Pa}$ discharges performed in a reactor internally covered with a-C:H. The concentrations of these species remain constant during the discharge, up to the complete film consumption [65] and, afterwards, $\mathrm{O}_{2}$ recovers its original concentration without discharge and $\mathrm{CO}, \mathrm{CO}_{2}$ and $\mathrm{H}_{2}$ disappear.
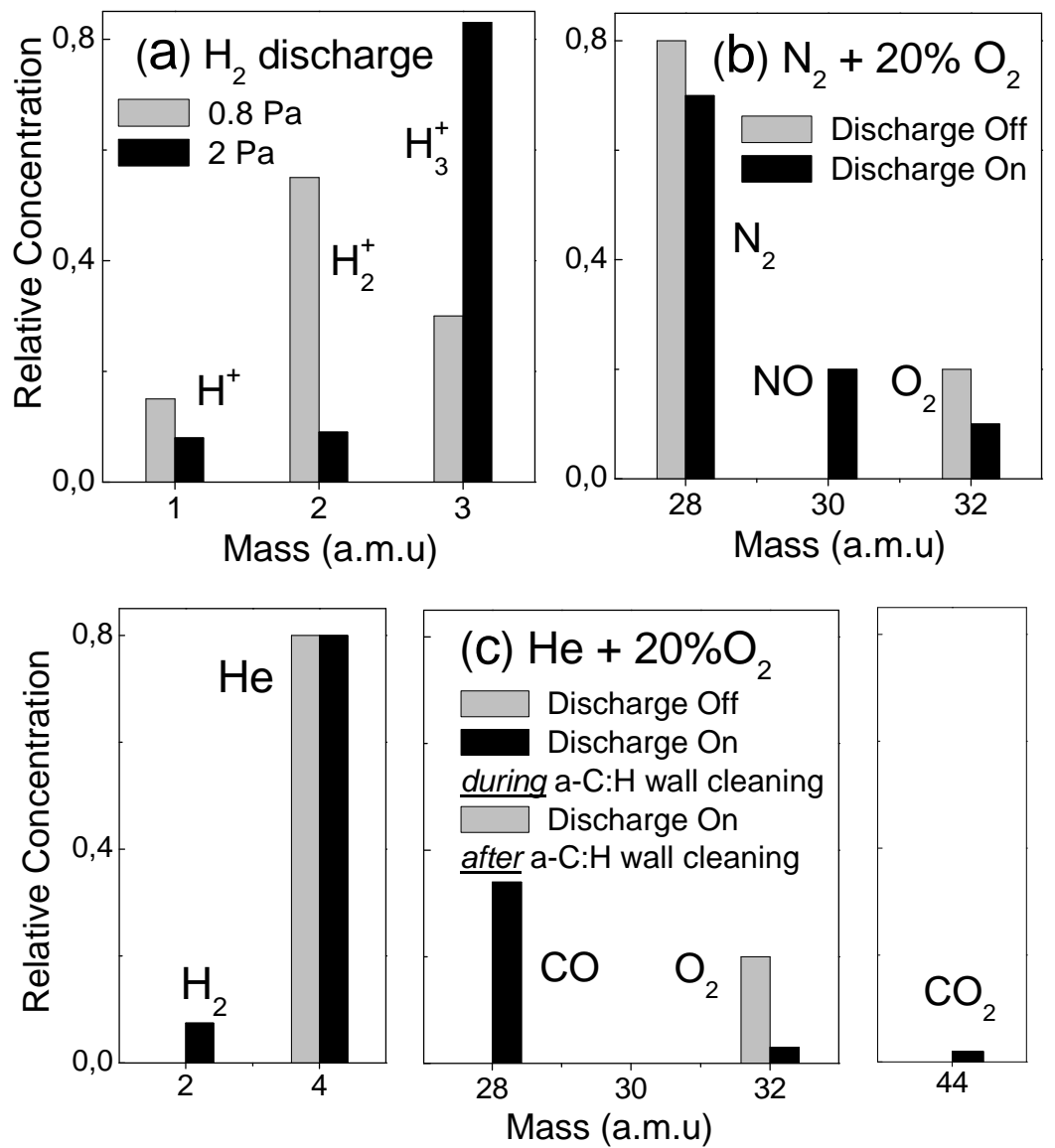

Figure 3. a) Relative ion concentrations in $\mathrm{H}_{2}$ discharges at 0.8 and $2 \mathrm{~Pa}$, showing large variations in $\mathrm{H}_{2}{ }^{+}$and $\mathrm{H}_{3}{ }^{+}$concentrations with pressure. b) $\mathrm{NO}$ generation and $\mathrm{N}_{2}+\mathrm{O}_{2}$ 
consumption in a $0.4 \mathrm{~Pa}$ air discharge. c) $\mathrm{O}_{2}$ decrease and $\mathrm{CO}+\mathrm{CO}_{2}+\mathrm{H}_{2}$ generation in a $\mathrm{He}+20 \% \mathrm{O}_{2}$ plasma reactor internally covered with a-C:H. In all the cases, a stainless steel hollow cathode reactor was used. The measurements were performed by mass spectrometry.

Glow discharges of mixtures containing light hydrocarbons like $\mathrm{CH}_{4}$ or $\mathrm{C}_{2} \mathrm{H}_{4}$ are extensively used to deposit a-C:H films [22,23,47], which, under appropriate growing conditions, exhibit diamond like properties [21]. $\mathrm{SiH}_{4}$ is commonly employed to deposit aSi:H films, widely used in microelectronics and solar cells. SiN films, useful as passivation coatings, are produced currently with $\mathrm{RF} \mathrm{SiH}_{4}+\mathrm{NH}_{3}$ discharges [66]. In all these processes, $\mathrm{H}_{2}$ and other products are generated, and marked depletions of the precursor gases are observed.

\section{Summary and outlook}

In the previous sections, the main processes involved in the chemistry of cold molecular plasmas generated in glow discharges have been commented on, from the initial steps leading to plasma formation, to the secondary gas phase and surface reactions. Subsequently, the most common plasma diagnostic techniques have been briefly introduced, including some recent and useful improvements, where sensitivity, dynamic range for species with concentrations spanning many orders of magnitude, and the need to determine their different energy distributions and roles in the plasma mechanisms are most essential. Some examples have illustrated the large differences found in the relevance of the various types of processes, although normally they act concurrently and provide these plasmas with their exceptional chemical reactivity at cold temperatures and their application capabilities.

In summary, the identification of the key plasma species remains essential to understand the basic mechanisms. Often, this is a difficult task, since most reactive species remain at the lowest concentrations. The relationships among the different processes and their dependence on plasma conditions are being currently investigated with combinations of 
experimental techniques, supported with kinetic models. These studies have produced interesting results, but also leave many open questions to address in the near future.

\section{Aknowledgments}

Some results presented here were obtained with financial support of the projects FIS201016455, CSD2009-00038 and FIS2007-61686 of the MICINN of Spain. J.M. Castillo, M.A. Moreno, J. Rodríguez and D. Pérez are gratefully recognized for technical assistance.

\section{Bibliography}

[1] Grill A. Cold Plasma in Materials Fabrication. New York: IEEE Press; 1993.

[2] Lieberman MA, Lichtenberg AJ. Principles of Plasma Discharges and Materials Processing. New Jersey: John Wiley \& Sons, Inc; 2005.

[3] Gordillo-Vázquez FJ, Herrero VJ, Tanarro I. Chem Vap Deposition 2007;13: 267.

[4] Hippler R, Kersten H, Schmidt M, Schoenbach KH , eds. Low temperature Plasmas: Fundamentals, Technologies and Techniques. Weinheim, Germany: Wiley-VCH; 2008.

[5] Federici G, Skinner CH, Brooks JN, Coad JP, Grisolia C, Haasz AA et al. Nucl. Fusion $2001 ; 41: 1967$.

[6] Herbst E. J Phys Chem A 2005;109:4017.

[7] Lochte-Holtgreven W. Plasma Diagnostics. Woodbury, NY: American Vacuum Society Classics, AIP; 1995.

[8] Méndez I, Gordillo-Vázquez FJ, Herrero VJ, TanarroI. J Phys Chem A 2006 ;110:6060.

[9] Itikawa I. Molecular Processes in Plasmas. Berlin: Springer-Verlag; 2007.

[10] Tanarro I, Herrero VJ. Plasma Sources Sci Technol (in press).

[11] M. Mozetic et al, J Nucl Mater 2007; 363-365:1457.

[12] Vesel A, Drenik A, Mozetic M, Balat-Pichelin M, Vacuum 2010; 84:969.

[13] Cvelbar U, Mozetic M, Poberaj I, Babib D, Ricard A, Thin Solid Films 2005; 475: 12. 
[14] Castillo M, Herrero VJ, Méndez I, Tanarro I. Plasma Sources Sci Techol 2004;13:343.

[15] Castillo M, Méndez I, Islyaikin AM, Herrero VJ, Tanarro I. J Phys Chem A 2005; 109:6255.

[16] Helden JH, Zijlmans RB, DC Schram, R Engeln. Plasma Sources Sci Techol 2009;18:025020.

[17] Gordiets B, Ferreira CM, Nahorny J, Pagnon D, Touzeau M, Vialle M. J Phys D: Appl Phys 1996;29:1021.

[18] Guerra V. IEEE Trans Plasma Sci 2007;35:1397.

[19] Kondo M, Fukawa M, Guo L, Matsuda A. J Non-Cryst Solids 2000;266-269:84.

[20] Kessels WMM, Smets AHM, Marra DC, Aydil ES, Schram DC, van de Sandem MCM, Thin Solid Films 2001;383:154.

[21] Jacob W, Thin Solid Films 1998;326:1.

[22] Bauer M, Schwarz-Selinger T, Jacob W, von Keudell A. J Appl Phys 2005;98:073302.

[23] Tabarés FL, Tafalla D, Tanarro I, Herrero VJ, Islyaikin AM. Vacuum 2004;73:161.

[24] Flewitt A J, Dyson AP, Robertson J, Milne W I. Thin Solid Films 2001;383:172.

[25] Sánchez M, editor. Nitrides and dilute nitrides: Growth, physics and devices. Kerala, India: Transworld Research; 2008.

[26] Kessels WMM, van Assche FJH, van den Oever PJ, van de Sanden MCM. J NonCrystalline Solids 2004;338-340:37.

[27] Benedikt J. J Phys D: Appl Phys 2010;43:043001

[28] Stillahn JM, Trevino KT, Fisher ER. Annu Rev Anal Chem 2008;1:261.

[29] Fantz U, Plasma Sources Sci Technol 2006;15:S137

[30] Thorne A, Litzén U, Johansson S. Spectrophysics, Principles and Applications. Berlin: Springer; 1999.

[31] Coburn JW, Chen M. J Appl Phys 1980;51:3134.

[32] Ma J, Ashfold MNR, Mankelevich YA. J Appl Phys 2009;105:043302. 
[33] Tanarro I, de los Arcos T, Domingo C, Herrero VJ, Sanz MM. Vacuum 2002; 64:457.

[34] Tanarro I, Sanz MM, Bermejo D, Domingo C, Santos J, Doménech JL. J Phys Chem1994;98:5862.

[35] Tanarro I, Sanz MM, Bermejo D, Domingo C, Santos J. J Chem Phys 1994;100:238.

[36] Demtröder W. Laser Spectroscopy: Basic Concepts and Instrumentation. Berlin: Springer-Verlag; 2003.

[37] Dawson PE, editor. Quadrupole Mass Spectrometry and its Applications. Woodbury: American Vacuum Society Classics, AIP Press; 1995.

[38] Tanarro I, Herrero VJ, Islyaikin AM, Méndez I, Tabarés FL, Tafalla D. J Phys Chem A 2007;111:9003.

[39] Kang HD, Preuss R, Schwartz-Selinger T, Dose V. J. Mass Spectrom. 2002;37: 748.

[40] Ferreira JA, Tabares FL. Plasma Sources Sci Techol 2009;18:034019.

[41] Morimoto T, Ansari SG, Yoneyama K, Nakajima T, Masuda A, Matsumura H et al. Jap J Appl Phys 2006;45:961.

[42] Sugai H, Toyoda H. J Vac Sci Technol A 2000;10:1113.

[43] Sanz MM, Abad L, Herrero VJ, Tanarro I. J Appl Phys 1992;71:5372.

[44] Singh H, Coburn JW, Graves DB. J Vac Sci Technol A 2000;18:299.

[45] Benedikt J, Eijkman DJ, Vandamme W, Agarwal S, van de Sanden MCM. Chem Phys Lett 2005;402:37.

[46] Herrero VJ, Islyiakin AM, Tanarro I. J Mass Spectrom 2008;43:1148.

[47] Tanarro I, Herrero VJ. Plasma Sources Sci Technol 2009;18:034007.

[48] Demidov VI, Ratynskaia SV, Rypdal K. Rev Sci Instrum 2002;73:3409.

[49] Crespo RM, Palop JIF, Hernandez MA, Ballesteros J. J Appl Phys 2003;94:4788

[50] Mozetic M, Cvelbar U, Vesel A, Ricard A, Babic D, Poberaj I. J Appl Phys 2005;97:103308

[51] Mozetic M, Vesel A, Cvelbar U, Ricard A. Plasma Chem Plasma Process 2006;26:103. 
[52] Stahl M, Trottenberg T, Kersten H. Rev Sci Instrum 2010;81:023504.

[53] Crespo RM, Palop JIF , Ballesteros J, Hernandez MA, Lucena-Polonio MV, Diaz-

Cabrera JM. Plasma Sources Sci Techol 2010;19:025012.

[54] Castillo M, Herrero VJ, Tanarro I. Plasma Sources Sci Techol 2002;11:368.

[55] de los Arcos T, Domingo C, Herrero VJ, Sanz MM, Schulz A, Tanarro I. J Phys Chem A 1998;102:6282.

[56] Malyshev MV, Donnelly VM. J Vac Sci Technol A 1997;15:550.

[57] Lavrov BP, Osiac M, Pipa AV, Röpcke J. Plasma Sources Sci Technol 2003;12: 576.

[58] Díez-Rojo T, Herrero VJ, Tanarro I, Tabarés FL, Tafalla D. Rev Sci Instrum 1997;68:1423.

[59] Tabarés FL, Tafalla D, Herrero VJ, Tanarro I. J Nuclear Materials 1997;241-243:1228.

[60] Guzman F, Tabares FL, Tafalla D, Cortes IG, Balbin R. J Nuclear Materials 2009; 39091:1127.

[61] Berndt J, Hong S, Kovacevic E, Stefanovic I, Winter J, Vacuum 2003;71:377.

[62] Geballe TR, Oka T. Nature 1996;384:334.

[63] Méndez I, Herrero VJ, Tanarro I. Phys Chem Chem Phys 2010;12:4239.

[64] Helden JH, van Wagemans W, Yagci G, Zijlmans RAB, Schram DC, Engeln RAH et al. J Appl Phys 2007;101:043305.

[65] Tanarro I, Ferreira JA, Herrero VJ, Tabarés FL, Gómez-Aleixandre C. J Nucl Mat 2009;390391:696.

[66] Romero MF, Sanz MM, Tanarro I, Jiménez A, Muñoz E. J Phys D: Appl Phys 2010; 43: 495202. 